\title{
Uso del microhábitat por el protozoo parásito Aggregata patagonica Sardella, Ré \& Timi, 2000 (Apicomplexa: Aggregatidae) en su hospedador definitivo, el pulpo Enteroctopus megalocyathus (Gould, 1852) (Cephalopoda: Octopodidae) en el sur de Chile
}

\author{
Microhabitat use by the protozoan parasite Aggregata patagonica Sardella, Ré \& Timi, \\ 2000 (Apicomplexa: Aggregatidae) in his definitive host Enteroctopus megalocyathus \\ (Gould, 1852) (Cephalopoda: Octopodidae) in southern Chile
}

\author{
CHRISTIAN M. IBÁÑEZ*, M. CECILIA PARDO-GANDARILLAS \\ \& MARIO GEORGE-NASCIMENTO
}

\begin{abstract}
Departamento de Ecología Costera, Facultad de Ciencias, Universidad Católica de la Santísima Concepción, Casilla 297, Concepción, Chile; *e-mail: cibanez@ucsc.cl
\end{abstract}

\begin{abstract}
RESUMEN
Los protozoos de la familia Aggregatidae requieren de dos hospedadores para completar su ciclo biológico: un crustáceo y un cefalópodo. En este estudio se busca evidenciar si existe un uso diferencial de microhábitats de las infrapoblaciones de Aggregata patagonica entre dos zonas del tracto digestivo de su hospedador definitivo, el pulpo Enteroctopus megalocyathus. Para ello, se examinaron 40 ejemplares de Ancud y 37 de Quellón, provenientes de las capturas artesanales de la Isla de Chiloé. El tamaño y la densidad de los ooquistes se cuantificaron en el ciego y el intestino del hospedador. Además, se efectuaron observaciones histológicas para determinar cuantitativamente la ocurrencia de los distintos estados de desarrollo del parásito. En Ancud el $80 \%$ de los pulpos estaban parasitados en el ciego y el intestino, mientras que en Quellón era el $100 \%$. Se determinaron cuatro estados de desarrollo (microgametos, cigotos, esporoquistes inmaduros y maduros). Los ooquistes de A. patagonica fueron más pequeños y abundantes en el ciego, pero la cobertura fue mayor en el intestino. Además, la densidad y cobertura aumenta en pocos meses entre una muestra y otra. Al parecer, A. patagonica no usaría a los dos microhábitats como lugares de preferencia para pasar los diferentes estados de desarrollo, sino más bien, y según las condiciones del microhábitat, en el intestino crecerían más los ooquistes pero en menos cantidad, ocupando una mayor superficie del microhábitat, contrario a lo que ocurre en el ciego. Por esto se sugiere que A. patagonica hace un uso diferencial del microhábitat, mediante dos estrategias distintas de historia de vida.
\end{abstract}

Palabras clave: microhábitat, Aggregata patagonica, Enteroctopus megalocyathus, ooquistes.

\section{ABSTRACT}

The protozoan parasites of the family Aggregatidae, require two hosts to complete their life cycle, a crustacean and a cephalopod. This research looks for evidence of differential microhabitat use of Aggregata patagonica infrapopulations between two zones of the digestive tract of his definitive host, the octopus Enteroctopus megalocyathus. Forty specimens from Ancud and 37 from Quellón coming from artisanal catch from Chiloé Island in southern Chile were examined. The size and density of the oocysts were quantified in the caecum and intestine of the host. Histological preparations were carried out to determine quantitatively the occurrence of the different development stages of the parasite. In Ancud, $80 \%$ of octopuses were parasited in the caecum and intestine, while in Quellón $100 \%$ had oocyst. Four development stages (microgametes, zygotes, immature and mature sporocysts) were recognized. The oocysts of Aggregata patagonica were smaller and more abundant in the caecum, but their coverage was higher in the intestine. Moreover, density and coverage increased in few months between samples. Apparently, Aggregata patagonica does not use the two microhabitats as preferential places to pass the different developmental stages, but rather, and according to the conditions of the microhabitat, the oocysts would grow more in the intestine albeit in small number, occupying a higher surface of the microhabitat, contrary to what happens in the caecum. This suggests that A. patagonica may have two distinct life history strategies.

Key words: microhabitat, Aggregata patagonica, Enteroctopus megalocyathus, oocysts. 


\section{INTRODUCCIÓN}

Los coccidios son endoparásitos intracelulares que poseen una estructura apical (el complejo apical) involucrada en la penetración de la célula hospedadora (MacFadden et al. 1997). Entre ellas, las especies de la familia Aggregatidae son parásitos de anélidos, moluscos y crustáceos marinos (Olsen 1974). Tienen un ciclo de vida que incluye dos hospedadores: la fase sexual (gamogonia y esporogonia) se desarrolla en el tracto digestivo de un cefalópodo, y la asexual (merogonia) ocurre en el de un crustáceo (Olsen 1974, Gestal et al. 1999a, 1999b, 2002a). Las especies del género Aggregata son los únicos coccidios que parasitan a los cefalópodos y se transmiten vía trófica (Olsen 1974). La especie mejor conocida es Aggregata eberthi (Labbé, 1895), que infecta a Sepia officinalis Linnaeus 1758 y al crustáceo Macropipus depurator (Linnaeus, 1758) en el Mediterráneo, Canal de la Mancha y el Mar del Norte (Gestal et al. 1999b, Gestal et al. 2002a, 2002b).

Sardella et al. (2000) describieron dos especies de Aggregata en pulpos patagónicos, A. valdessensis Sardella Ré \& Timi, 2000 en Octopus tehuelchus d' Orbigny 1834 y A. patagonica Sardella Ré \& Timi, 2000 en Enteroctopus megalocyathus (Gould, 1852), con prevalencias de 72 y $77 \%$, respectivamente. En estos pulpos, los coccidios se reconocen macroscópicamente en las paredes del buche, ciego e intestino como quistes blancos en estado de esporogonia (Sardella \& Ré 1990, Sardella et al. 2000). Incluso hay registros aislados de infecciones masivas de todos los órganos que componen el sistema digestivo (exceptuando el estómago) (Estévez et al. 1996). Sin embargo, Gestal (2000) encontró que A. octopiana (Schneider, 1875) infecta a los pulpos en las zonas del tracto digestivo desprovistas de cutícula, es decir, en el ciego e intestino. El ciego es un órgano de absorción y digestión activa, en el cual los alimentos permanecen largo tiempo (BoucaudCamou \& Boucher-Rodoni 1983) por la mayor superficie de absorción, consecuencia de los numerosos pliegues contiguos y el enrollamiento en espiral de sus paredes, que lo hace un sitio preferido para la invasión de los merozoitos (Sardella \& Ré 1990, Estévez et al. 1996). Por otro lado, el intestino es solo un ducto de descarga de los desechos alimenticios (Boucaud-Camou \& Boucher-Rodoni 1983). Estas diferencias morfofuncionales de ambos microhábitat podrían obligar al parásito a desarrollar distintos medios de ocupación considerando esta utilización como la forma en que el parásito se desarrolla y cubre los microhábitats.

Los estudios parasitológicos en este grupo de protozoos han sido extensivamente descriptivos en cuanto a la sistemática de las especies, sus ciclos de vida y los daños causados por el parásito al hospedador (Olsen 1974, Sardella \& Ré 1990, Estévez et al. 1996, Sardella \& Martorelli 1997, Gestal et al. 1999a, 1999b, Vidal \& Haimovici 1999, Gestal 2000, Gestal et al. 2000, Sardella et al. 2000, Gestal et al. 2002a, 2002b, 2002c, 2002d), no existiendo trabajos con un enfoque ecológico y sobre todo en relación con las variaciones infrapoblacionales.

\section{MATERIALES Y MÉTODOS}

Se examinaron 40 ejemplares del pulpo $E$. megalocyathus capturados mediante buceo semiautónomo por buzos artesanales en los alrededores de la localidad de Ancud ( $41^{\circ} 47^{\prime} \mathrm{S}$, $74^{\circ} 28^{\prime}$ O) en junio de 2000 , y 37 de la localidad de Quellón ( $\left.43^{\circ} 27^{\prime} \mathrm{S}, 74^{\circ} 47^{\prime} \mathrm{O}\right)$, en octubre de 2000, Chiloé, X Región, Chile. En el laboratorio, luego de descongelados, a cada ejemplar se le midió la longitud dorsal del manto (LDM en cm, según Roper \& Voss 1983). Mediante disección de cada ejemplar, se extrajo el tracto digestivo, que fueron posteriormente guardados y preservados en congeladores a $-15{ }^{\circ} \mathrm{C}$ hasta su examen. Luego de descongelados los tractos digestivos, solo se inspeccionó el ciego e intestino, debido a que son los órganos que presentan la mayor presencia y densidad de ooquistes (Sardella \& Ré 1990), a los cuales se les midió el área. Las áreas del ciego y el intestino fueron calculadas como el área de una elipse, y la de un rectángulo, respectivamente. Al mismo tiempo, se realizaron disecciones solo del ciego y el intestino, para determinar la presencia de ooquistes de A. patagonica. En el intestino y el ciego la cuantificación de los ooquistes se realizó por medio de un muestreo dirigido, a través de tres cuadrantes de un centímetro 
cuadrado colocados en la región proximal, media y distal de cada microhábitat. Conjuntamente, 20 ooquistes (cuando fue posible) fueron medidos en su largo y ancho $(\mathrm{mm})$ en el ciego e intestino, a través de una reglilla ocular en un microscopio estereoscópico con aumento de 20x y posteriormente guardados en formalina al $10 \%$ en frascos debidamente rotulados. Luego se calculó el área de los ooquistes $\left(\mathrm{mm}^{2}\right)$ por medio de la superficie de una elipse, debido a que estos son más largos que anchos, considerándola como el tamaño corporal de los ooquistes para tener una aproximación del área que ocupan en el microhábitat.

Para evaluar la ocupación en los dos microhábitats, se procedió a calcular la cobertura de los ooquistes en cada órgano para cada ejemplar, expresado, porcentualmente, por la siguiente expresión:

$$
C_{j}=\frac{N_{j} \times \frac{1}{n} \times \sum_{i=1}^{n} T_{i}}{A_{j}}
$$

donde $\mathrm{N}_{\mathrm{j}}$ es el número de ooquistes por $\mathrm{cm}^{2}$ en el microhábitat del hospedador $\mathrm{j}$; $\mathrm{n}$ el número de ooquistes medidos; $\mathrm{T}_{\mathrm{i}}$ el tamaño de los ooquistes $\mathrm{i}$; y $\mathrm{A}_{\mathrm{j}}$ el área promedio de los microhábitats $\left(\mathrm{cm}^{2}\right)$ en el hospedador $\mathrm{j}$.

Quince ejemplares de la localidad de Quellón fueron seleccionados para realizar cortes histológicos de tejido con ooquistes del ciego e intestino, los cuales fueron fijados en formalina al $10 \%$, deshidratados en una batería de etanol graduada, y embebidos en parafina sólida. Posteriormente, secciones de $5 \mu \mathrm{m}$ fueron teñidas en hematoxilina-eosina (Culling et al. 1985). Los cortes histológicos se fotografiaron a través de un microscopio óptico conectado a un monitor y computador, con el objeto de determinar la especie de Aggregata, identificar los estados del ciclo biológico en el ciego e intestino del hospedador según Sardella \& Ré (1990) y Sardella et al. (2000), y medir el diámetro de los esporoquistes, para compararlos entre los microhábitats.

La parasitosis fue caracterizada mediante la prevalencia (número de pulpos infectados del total de ejemplares analizados; Margolis et al. 1982), y la densidad (número de ooquistes por $\mathrm{cm}^{2}$; Bush et al. 1997).
Las distribuciones de frecuencia del tamaño de los ooquistes fueron comparadas entre microhábitats mediante la prueba de Kolmogorov-Smirnov para dos muestras (Zar 1984).

La significancia estadística de las variaciones de la densidad y cobertura de los ooquistes entre el ciego e intestino y entre los muestreos fue evaluada mediante un análisis de varianza de dos vías previa transformación de los datos a ranking (Zar 1984).

Para comparar la frecuencia de ocurrencia de los estados de desarrollo del parásito en los distintos microhábitats se realizó una tabla de contingencia de máxima probabilidad (test de G), evaluando su significancia estadística mediante Chi-cuadrado (Zar 1984).

El diámetro de los esporoquites del ciego e intestino fueron comparados mediante una prueba de pares emparejados de Wilcoxon (Zar 1984).

\section{RESULTADOS}

Macroscópicamente, el estado de esporogonia es la única etapa que se distinguió como ooquistes de distintos tamaños, de forma esférica y de color blanco en el ciego e intestino.

Microscópicamente, de los estados sexuales descritos (Sardella et al. 2000), los cuales solamente pueden ser identificados a través de cortes histológicos de los órganos infectados, solo fueron visualizados el microgamético, cigótico, esporoquistes inmaduros y maduros. Estos últimos, en el interior de la cápsula contienen ocho esporozoitos vermiformes uninucleados (Sardella et al. 2000).

La longitud dorsal del manto de los pulpos fluctuó entre 15 y $22 \mathrm{~cm}$ en Ancud (19,3 $\pm 2,24$ $\mathrm{cm})$. Casi todos presentaron infección con el coccidio A. patagonica ( $80 \%$ ), en el estado de ooquistes. Los pulpos que no presentaron ooquistes, los habían tenido anteriormente, ya que tenían las paredes del ciego destruidas por la liberación de los esporoquistes. Los ooquistes del intestino resultaron ser más grandes que los ooquistes del ciego (Tabla 1, Fig. 1A y 1B). Al comparar sus distribuciones de frecuencias en cada microhábitat, se encontró que estas resultaron ser distintas $\left(D_{\max }\right.$ $=0,68, \mathrm{P}<0,001)$ (Fig. 2A). La longitud dorsal 
del manto de los pulpos fluctuó entre 12 y 23 $\mathrm{cm}$ en Quellón $(16,9 \pm 2,88 \mathrm{~cm})$. Todos presentaron infección con el coccidio Aggregata patagonica (100 \%), en los estados: microgamético, cigótico y esporoquistes maduros (esporozoitos) e inmaduros, en el ciego e intestino. Los ooquistes del intestino resultaron más grandes que los ooquistes del ciego (Tabla 1, Fig. 2B), y al comparar sus distribuciones de frecuencias de estos en cada microhábitat, se encontró que eran distintas $\left(\mathrm{D}_{\max }=0,88, \mathrm{P}<0,001\right)$ (Fig. 2B). Algunos ejemplares de Quellón presentaron ooquistes de gran tamaño en la unión del ciego con el intestino, lo que se puede apreciar en las distribuciones de frecuencias de tamaños, ya que existe una superposición en el rango de tamaños con los ooquistes del intestino (Fig. 2B). El área de los microhábitats fue similar con promedios de 25,6 $\pm 6,3 \mathrm{~cm}^{2}$ en el ciego y $27,3 \pm 8,1 \mathrm{~cm}^{2}$ en el intestino.

En ambos muestreos la densidad de ooquistes resultó mayor en el ciego (Tabla 1) (Fig. 3), mientras que la cobertura de los ooquistes en ambos muestreos, es mayor en el intestino (Tabla 1, Fig. 4). Esto es así porque los ooquistes del intestino ocupan una mayor área que los del ciego a pesar de que el área del ciego, considerando los pliegues, sería mayor que la del intestino.

Al comparar las densidades y coberturas de ooquistes del ciego e intestino entre muestreos, se encontró que aumentaron en ambos microhábitats desde junio (Ancud) a octubre (Quellón) (Fig. 3 y 4). Las densidades en el ciego e intestino resultaron significativamente distintas entre estos microhábitats $\left(\mathrm{F}_{1,137}=4,43\right.$, $\mathrm{P}=0,0371)$ y entre las fechas de muestreo $\left(\mathrm{F}_{1,137}=55, \mathrm{P}=0,0001\right)$, siendo el factor muestreo de mayor importancia, y la interacción de estos factores no fue significativa $\left(\mathrm{F}_{1,137}=\right.$ $1,87, \mathrm{P}=0,1734)$. Igualmente las coberturas son distintas entre microhábitat $\left(\mathrm{F}_{1,137}=94,37, \mathrm{P}=\right.$ $0,0001)$ y muestreos $\left(F_{1,137}=71,45, P=0,0001\right)$, pero no en cambio el factor más relevante en este caso son los microhábitat. Igualmente, la interacción de los factores no resultó ser distinta $\left(\mathrm{F}_{1,137}=3,7, \mathrm{P}=0,0565\right)$.

TABLA 1

Tamaño de los ooquistes $\left(\mathrm{mm}^{2}\right)$, densidad (número $\mathrm{cm}^{-2}$ ) y cobertura $(\%)$ de Aggregata patagonica en el ciego e intestino de Enteroctopus megalocyathus en dos localidades de la Isla de Chiloé

Oocyst size $\left(\mathrm{mm}^{2}\right)$, density (número $\mathrm{cm}^{-2}$ ) and cover $(\%)$ of Aggregata patagonica in caecum and intestine of Enteroctopus megalocyathus at two localities from Chiloé Island

\begin{tabular}{|c|c|c|c|c|c|}
\hline & $\mathrm{N}$ & Promedio & Mínimo & Máximo & $\mathrm{DE}$ \\
\hline Ciego & \multicolumn{5}{|c|}{ Ancud (junio) } \\
\hline Tamaño & 282 & 0,12 & 0,03 & 0,44 & 0,07 \\
\hline Densidad & 32 & 15,46 & 0 & 89,00 & 21,00 \\
\hline Cobertura & 32 & 0,64 & 0 & 2,54 & 0,75 \\
\hline \multicolumn{6}{|l|}{ Intestino } \\
\hline Tamaño & 377 & 1,67 & 0,13 & 4,71 & 0,85 \\
\hline Densidad & 32 & 4,68 & 0,33 & 15,67 & 3,96 \\
\hline Cobertur & 32 & 2,76 & 0,09 & 10,14 & 2,80 \\
\hline Ciego & \multicolumn{5}{|c|}{ Quellón (octubre) } \\
\hline Tamaño & 340 & 0,44 & 0,11 & 2,43 & 0,32 \\
\hline Densidad & 37 & 34,12 & 1,00 & 124,33 & 30,64 \\
\hline Cobertura & 37 & 2,04 & 0,03 & 6,52 & 1,69 \\
\hline \multicolumn{6}{|l|}{ Intestino } \\
\hline Tamaño & 752 & 1,50 & 0,13 & 4,85 & 0,70 \\
\hline Densidad & 37 & 24,96 & 2,33 & 65,67 & 18,13 \\
\hline Cobertura & 37 & 12,14 & 1,59 & 41,24 & 9,24 \\
\hline
\end{tabular}


(A)

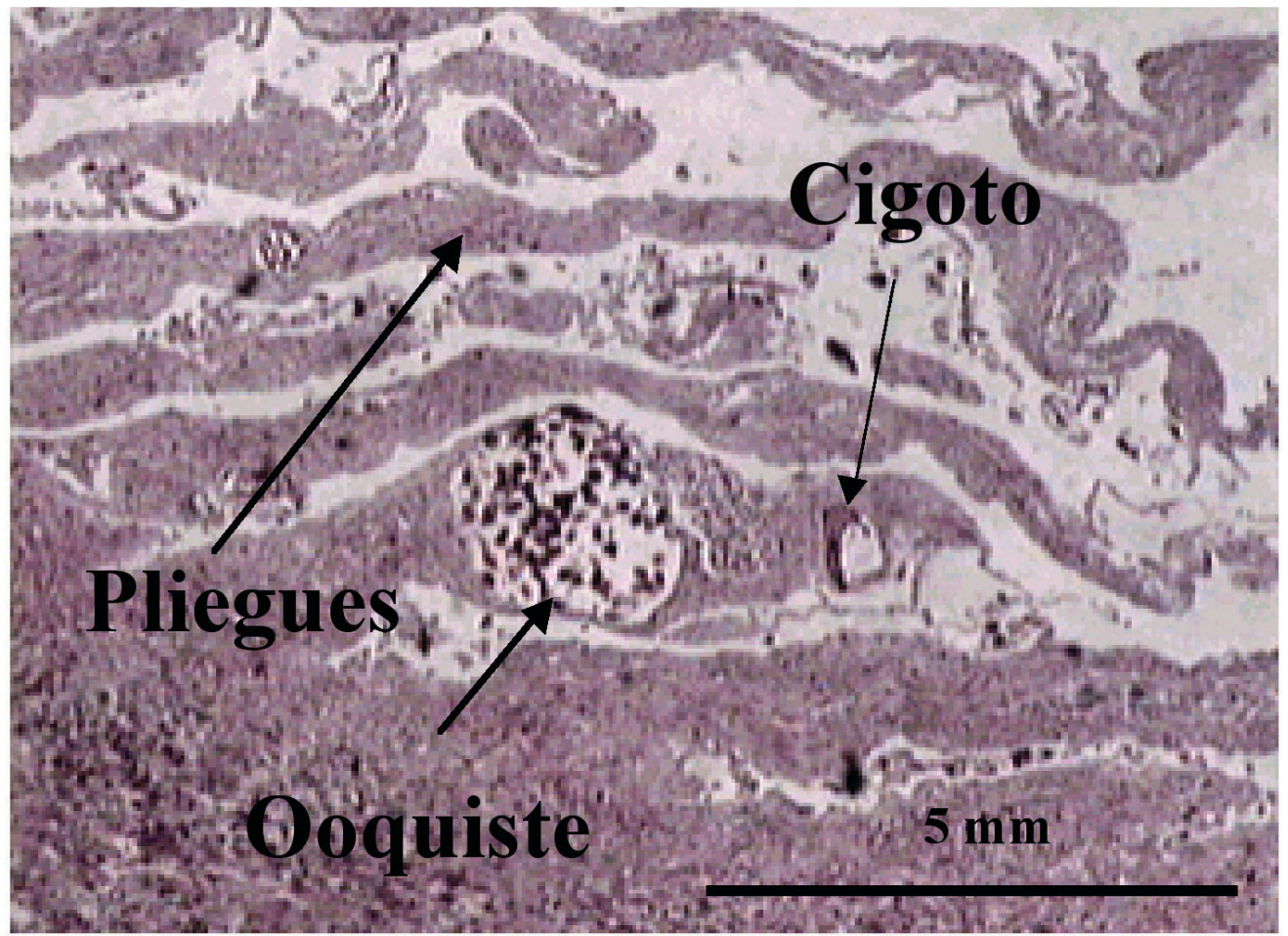

(B)

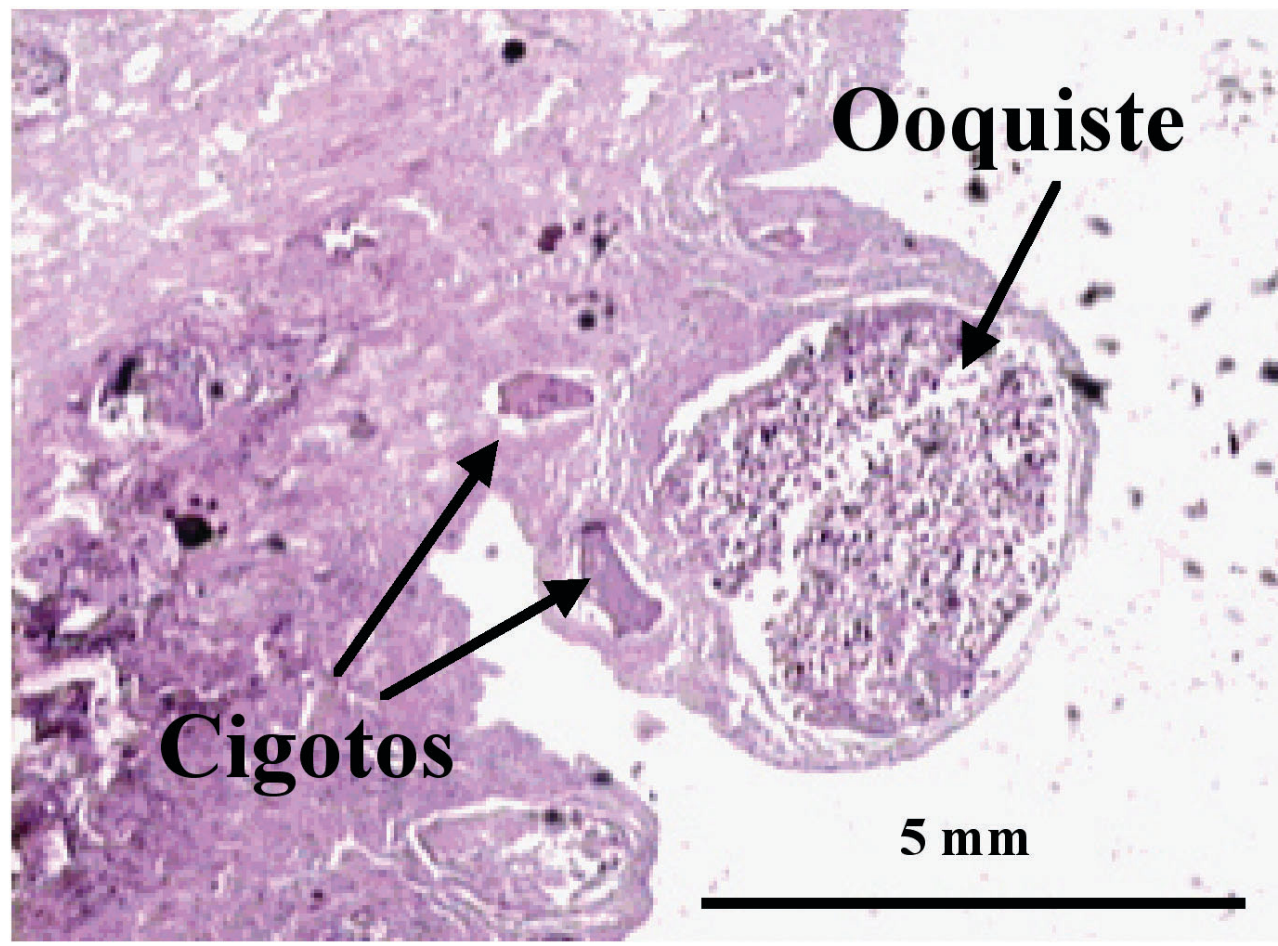

Fig. 1: Fotomicrografías de los estados de infección de Aggregata patagonica en el ciego (A) e intestino (B) de Enteroctopus megalocyathus.

Photomicrography of infection stage of Aggregata patagonica in caecum (A) and intestine (B) of Enteroctopus megalocyathus. 
De las muestras histológicas de Quellón, tanto en el ciego como en el intestino se encontraron los mismos estados de desarrollo del parásito (exceptuando a dos ejemplares), y con una ocurrencia no muy distinta entre ellas $\left(\chi^{2}=2,49\right.$, g.l. $\left.=3, P=0,47\right)$. Esto se puede observar en las fotomicrografías (Fig. 1A y 1B). Los diámetros de los esporoquistes resultaron similares entre el ciego y el intestino $(\mathrm{Z}=0,46, \mathrm{n}=90, \mathrm{P}=0,64)$, con promedios de $15,2 \pm 6,6 \quad \mu \mathrm{m} \quad$ y $14,6 \pm 2,7 \quad \mu \mathrm{m}$, respectivamente.

(A)

Ciego $(n=282)$ Intestino $(\mathbf{n}=\mathbf{3 7 7})$

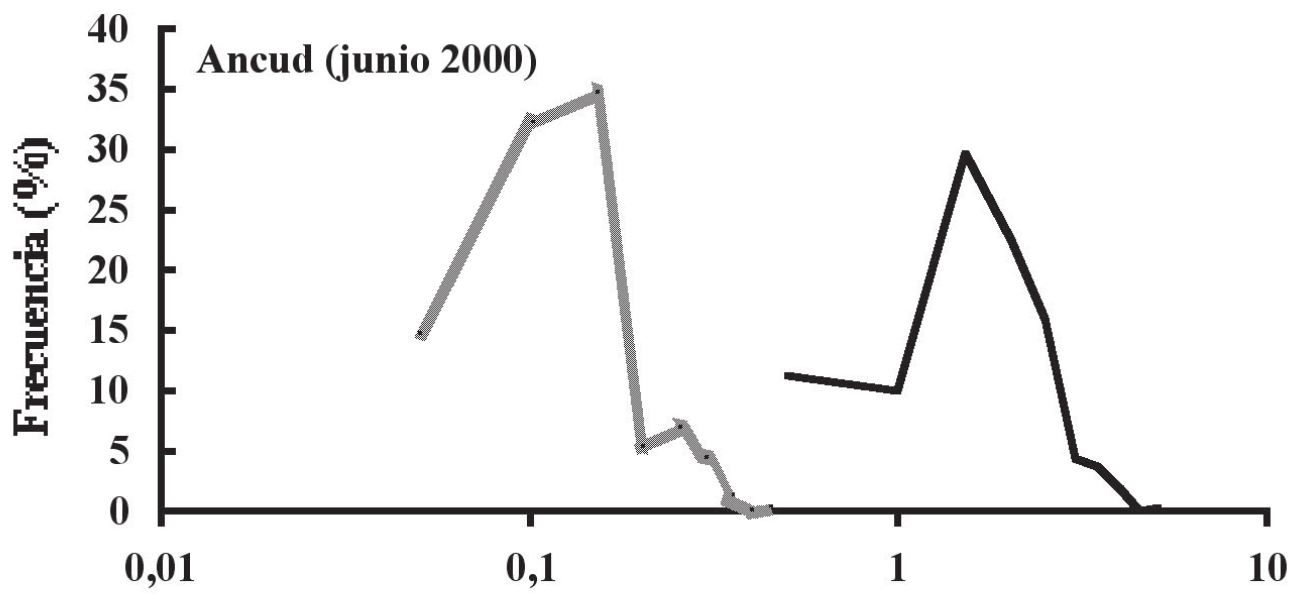

Tamaño ooquistes $\left(\mathrm{mm}^{2}\right)$

(B)
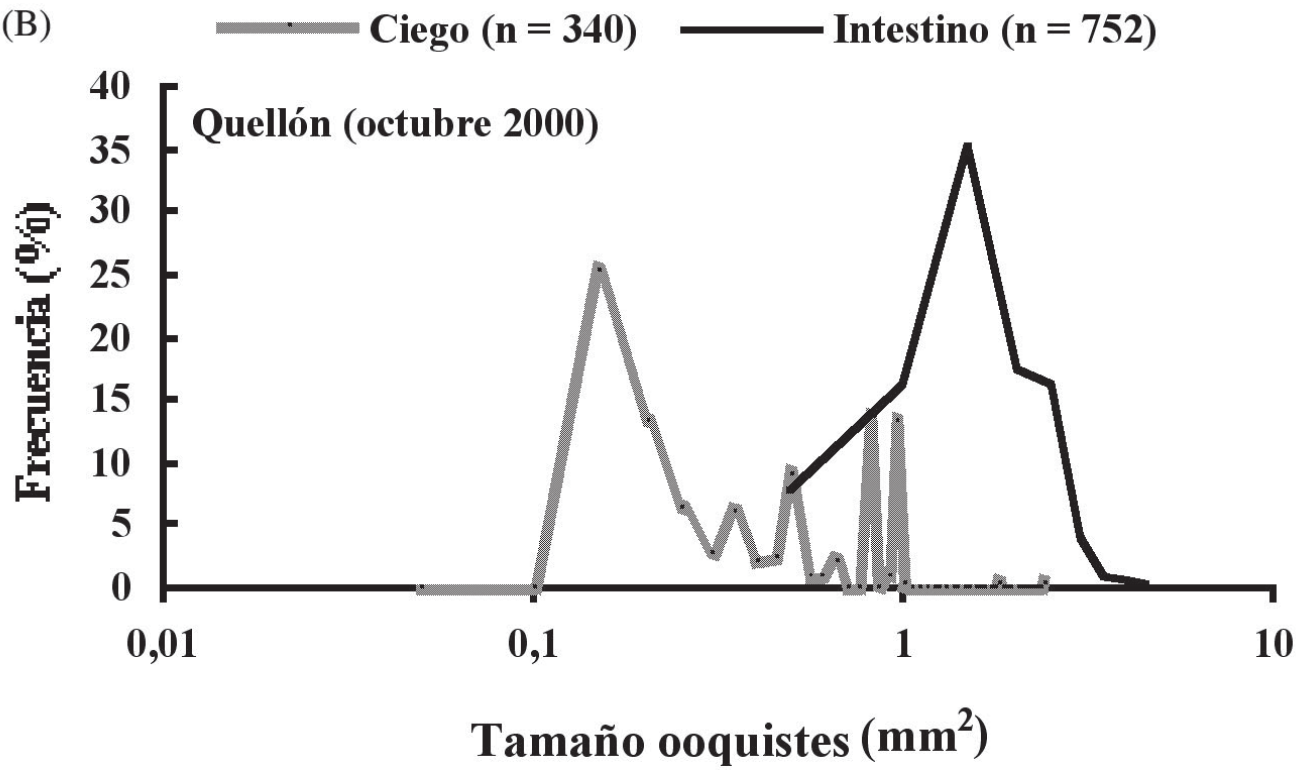

Fig. 2: Distribución de frecuencias de tamaños de los ooquistes de Aggregata patagonica en el ciego e intestino de Enteroctopus megalocyathus en Ancud (A) y Quellón (B). Los tamaños de ooquistes se presentan en escala logarítmica.

Size frequency distributions of oocyst of Aggregata patagonica in caecum and intestine of Enteroctopus megalocyathus at Ancud (A) and Quellón (B). Oocyst sizes are in logarithmic scale. 
口 Ancud (junio 2000) Q Quellón (octubre 2000)

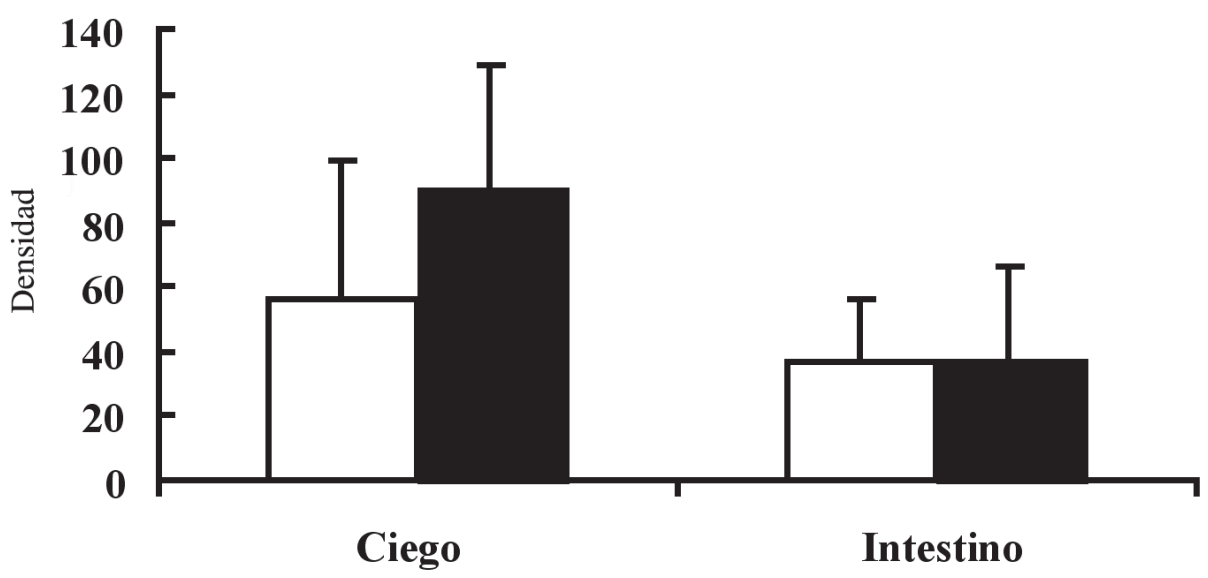

Microhábitat

Fig. 3: Densidad (ranking) de los ooquistes de Aggregata patagonica en dos microhábitats del tracto digestivo de Enteroctopus megalocyathus. Las barras representan la desviación estándar.

Density of the oocysts of Aggregata patagonica in two microhabitats of the digestive tract of Enteroctopus megalocyathus. Bars represent the standard deviation.

口Ancud (junio 2000) Q Quellón (octubre 2000)

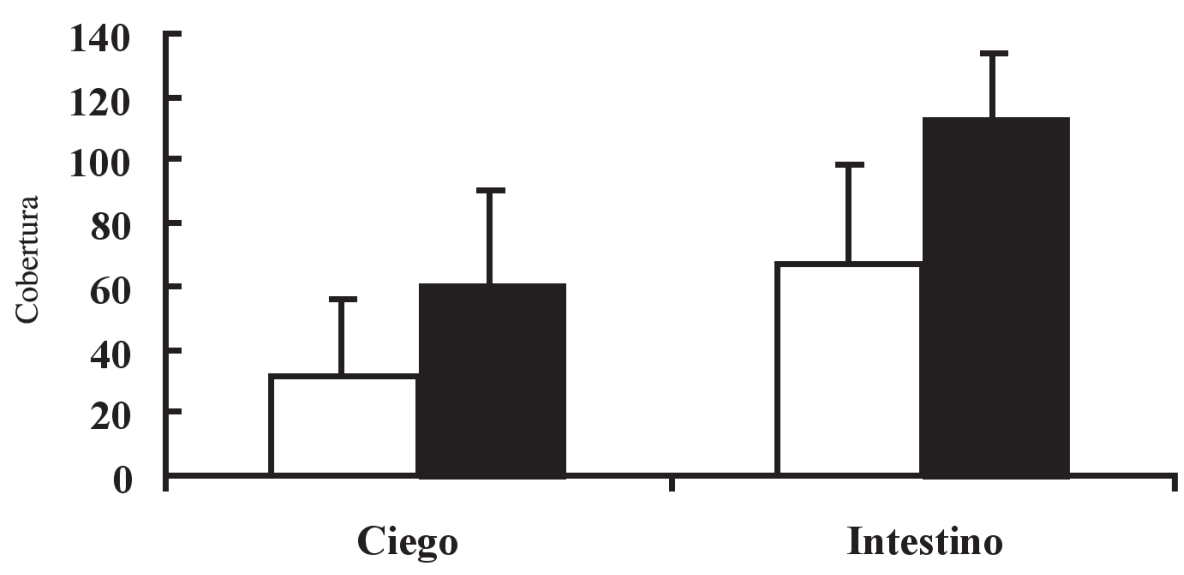

Microhábitat

Fig. 4: Cobertura (ranking) de los ooquistes de Aggregata patagonica en dos microhábitats del tracto digestivo de Enteroctopus megalocyathus. Las barras representan la desviación estándar.

Cover of the oocysts of Aggregata patagonica in two microhabitats of the digestive tract of Enteroctopus megalocyathus. Bars represent the standard deviation. 


\section{DISCUSIÓN}

Aggregata patagonica infecta en la etapa sexual al ciego e intestino de E. megalocyathus, su hospedador definitivo, sin mostrar cuantitativamente una preferencia por dichos microhábitats, en cuanto a la ocurrencia de los estados del desarrollo.

Los ooquistes en el ciego son más pequeños y presentan una mayor densidad que en el intestino, característica muy distintiva en este trabajo, documentado además en Argentina por Norma Sardella en el mismo sistema hospedador-parásito (comunicación personal 2002). El ciego está conformado morfológicamente por numerosos pliegues delgados muy contiguos que aumentan la superficie (Fig. 1A), los cuales son considerados como limitantes para el desarrollo de un mayor tamaño, pero no para la densidad de los ooquistes. Además, el ciego es un órgano de absorción que presenta variaciones del pH durante la digestión (Lipinski 1990), lo que podría ser otro factor limitante para el crecimiento de los ooquistes. En contraste, el cambio de la textura del tubo digestivo en una superficie levemente rugosa desde la conexión con el ciego hacia el resto del intestino (Fig. 1B), serían características microambientales que favorecen un mayor tamaño, pero a la vez menor densidad de ooquistes. Sin embargo, la superficie que cubren los ooquistes es mayor en el intestino, debido a su mayor tamaño. Así, tanto el tamaño como la densidad y cobertura diferencial de los ooquistes entre los órganos, serían consecuencia de las características morfológicas y funcionales distintivas de los microhábitat que fuerzan al parásito a usarlos diferencialmente. Debido a que el tamaño diferencial de los ooquistes entre los distintos microhábitats corresponde a los mismos estados reproductivos, se sugiere que estos se entienden como dos estrategias de historia de vida distintas. Dado que los esporoquistes son casi del mismo tamaño en ambos microhábitats, la segunda estrategia constituiría una mayor producción de esporoquistes. Según Boucaud-Camou \& Boucher-Rodoni (1983) el buche y estómago de los pulpos presenta en su interior una bolsa con una cutícula muy dura, lo que sugirió a Sardella \& Ré (1990) el impedimento del asentamiento de los merozoitos. Sin embargo, hay registros en Octopus vulgaris Cuvier 1797 de infecciones masivas, presentando enquistamiento en forma generalizada en varios órganos (Gestal et al. 1996, Gestal et al. 2002c). Al parecer los merozoitos de Aggregata patagonica que ingresan a este hospedador se asientan y desarrollan parte de su ciclo biológico en zonas distintivas del tracto digestivo. No obstante, en casos en que aumenta el consumo de presas infectadas, y así las reinfecciones por este parásito, se produciría una reducción del espacio en los microhábitat que frecuentemente son ocupados.

Las altas densidades de ooquistes probablemente actúan sobre el hospedador reduciendo el área de absorción del ciego, generando como consecuencia una disminución de los componentes de la adecuación biológica por parte del hospedador, similar a lo reportado por Gestal et al. (1999b, 2002d) y Sardella \& Ré (1990). Cuando la infección es generalizada puede incluso provocar graves daños fisiológicos, como trastornos de absorción en $O$. vulgaris resultado de la infección parasitaria de A. octopiana (Gestal et al. 2002d). Además, los pulpos disminuyen sus tasas de crecimiento, el tamaño máximo, y su esperanza de vida (Gestal et al. 1999b). Simultáneamente, A. octopiana causa una hipertrofia de las células hospedadoras con un desplazamiento del núcleo, inflamación, fagocitosis, ulceración y destrucción de la arquitectura de los órganos (Gestal et al. 2002c). No obstante, las infecciones por coccidios no son la primera causa de muerte en las poblaciones naturales de pulpos (Gestal et al. 1999b).

Muchos estudios indican que Aggregata spp. parasita a crustáceos braquiuros como hospedadores intermediarios. Sin embargo, Sardella \& Martorelli (1997) han encontrado estos coccidios en crustáceos del suborden Natantia (Pleoticus muelleri (Bate 1888)y Artemesia longinaris Bate 1888), los que en aguas patagónicas (Argentina) podrían ser los hospedadores intermediarios que transmitirían el parásito a E. megalocyathus y $O$. tehuelchus. Por otro lado, en las costas de Chiloé (Chile), megalocyathus se alimenta de crustáceos braquiuros como Homalaspis plana (MilneEwards 1834), Cancer setosus (Molina 1782), Cancer coronatus (Molina 1782) y anomuros 
como Munida subrugosa (White 1847), y Petrolisthes sp. (Ibáñez et al. 2001²). Estos podrían ser hospedadores intermediarios de Aggregata patagonica en esta zona geográfica. Se sugiere que la abundancia de ooquistes está altamente relacionada con los hábitos alimenticios del hospedador, y que es dependiente de la frecuencia de consumo de crustáceos infectados. Además, ya que estos parásitos son muy específicos en el caso de los cefalópodos, pero no así en los hospedadores intermediarios (Olsen 1974), la baja especificidad del parásito por el hospedador intermediario, al desarrollarse en varias especies de crustáceos, aumenta la probabilidad de transmisión.

Finalmente hay que considerar que tanto la época del año como la localidad de estudio podrían ser factores importantes en el estudio de los microparásitos de una población de pulpos, como también la tasa de consumo de presas que podrían ser transmisoras de los parásitos. Sin embargo, este estudio solo analizó una pequeña muestra en un periodo de tiempo muy breve. La mayor densidad de ooquistes en primavera (octubre) coincide con lo reportado por Sardella \& Ré (1990), y según estos autores se debe al aumento de la temperatura del mar, que consecuentemente induce un incremento en las tasas de alimentación y crecimiento de los pulpos. No obstante, recientemente González et al. (2003) sugieren que los factores ambientales no son tan determinantes del riesgo del parasitismo en los cefalópodos, pero sí lo son características ecológicas como la conducta, dieta, historia de vida, tamaño corporal, movilidad y tipo de hábitat.

\section{AGRADECIMIENTOS}

Agradecemos la ayuda de Javier Chong, que proporcionó las muestras del proyecto FIP 9920 "Estudio biológico pesquero del recurso pulpo en las regiones X y XI". Además a Edith Montero por su valiosa ayuda en las

IBÁÑEZ C, N CORTÉS \& J CHONG (2001) Aspectos trofodinámicos del pulpo. Enteroctopus megalocyathus en el Pacífico suroriental. Resúmenes XXI Congreso Ciencias del Mar, Viña del Mar, Chile. preparaciones histológicas, Camino Gestal de la Universidad de Vigo, España, por la literatura que proporcionó, y a Norma Sardella de la Universidad de la Plata, Argentina, por sus comentarios y ayuda en la determinación tanto taxonómica como del ciclo biológico del parásito, y finalmente a Luis Cubillos por su aporte en el cálculo de la cobertura.

\section{LITERATURA CITADA}

BOUCAUD-CAMOU E \& R BOUCHER-RODONI (1983) Feeding and digestion in Cephalopods, En: RussellHunter WD (ed) The Mollusca, Volume 2: Physiology of Mollusca: 149-190. Academic Press, New York, New York, USA.

BUSH AO, KD LAFFERTY, JM LOTZ \& AW SHOSTAK (1997) Parasitology meets ecology on its own terms: Margolis et al. revisited. Journal of Parasitology 83: 575-583.

CULLING CFA, RT ALLISON \& W T BARR (1985) Cellular pathology techniques. Butterworth \& Co. Ltd. London. United Kingdom. 344 pp.

ESTÉVEZ J, S PASCUAL, C GESTAL, M SOTO, H RODRÍGUEZ \& C ARIAS (1996) Aggregata octopiana (Apicomplexa: Aggregatidae) from Octopus vulgaris off NW Spain. Diseases of Aquatic Organisms 27: 227-231.

GESTAL C, E ABOLLO \& S PASCUAL (1999a) Evaluation of a method for isolation and purification of sporocysts of the cephalopod coccidian parasite Aggregata Frenzel, 1885 (Apicomplexa: Aggregatidae). Iberus 17: 115-121.

GESTAL C, S PASCUAL, L CORRAL \& C AZEVEDO (1999b) Ultrastructural aspects of the sporogony of Aggregata octopiana (Apicomplexa, Aggregatidae), a coccidian parasite of Octopus vulgaris (Mollusca, Cephalopoda) from NE Atlantic Coast. European Journal of Protistology 35: 417-425.

GESTAL C (2000) Epidemiología y patología de las coccidiosis en cefalópodos. Tesis Doctoral, Universidad de Vigo, Vigo, España. 157 pp.

GESTAL C, A GUERRA, E ABOLLO \& S PASCUAL (2000) Aggregata sagittata n. sp. (Apicomplexa: Aggregatidae), a coccidian parasite from the european flying squid Todarodes sagittatus (Mollusca: Cephalopoda). Systematic Parasitology 47: 203-206.

GESTAL C, A GUERRA, S PASCUAL \& C AZEVEDO (2002a) On the life cycle of Aggregata eberthi and observations on Aggregata octopiana (Apicomplexa, Aggregatidae) from Galicia (NE Atlantic). European Journal of Protistology 37: 427435.

GESTAL C, C SERRA, A GUERRA \& S PASCUAL (2002b) Scratching the sporocyst surface: characterisation of European Aggregata species by atomic microscopy. Parasitology Research 88: 242246.

GESTAL C, E ABOLLO \& S PASCUAL (2002c) Observations on associated histopatology with Aggregata octopiana infection (Protista: Apicomplexa) in Octopus vulgaris. Diseases of Aquatic Organisms 50: 45-49.

GESTAL C, MP DE LA CADENA \& S PASCUAL (2002d) Malabsortion syndrome observed in the 
common octopus Octopus vulgaris infected with Aggregata octopiana (Protista: Apicomplexa). Diseases of Aquatic Organisms 51: 61-65.

GONZÁLEZ AF, S PASCUAL, C GESTAL, E ABOLLO \& A GUERRA (2003) What makes a cephalopod a suitable host for parasite? The case of Galician waters. Fisheries Research 60: 177-183.

LIPINSKI MR (1990) Changes in $\mathrm{pH}$ in the caecum of Loligo vulgaris reynaudii during digestion. South African Journal of Marine Science 9: 43-51.

MACFADDEN GI, RF WALLER, ME REITH \& N LANG-UNNASCH (1997) Plastids in apicomplexan parasites. Plant Systematics and Evolution 11: 261287.

MARGOLIS L, GW ESCH, JC HOLMES, AM KURIS \& GA SCHAD (1982) The use of ecological terms in parasitology. Journal of Parasitology 68: 131-133.

OLSEN OW (1974) Animal parasites, their life cycles and ecology. Dover Publications, Inc., New York, New York, USA. 562 pp.

ROPER CF \& GL VOSS (1983) Guidelines for taxonomic descriptions of Cephalopods species. Memoirs of the National Museum of Victoria (Australia) 44: 49-63.

Editor Asociado: Rodrigo Medel

Recibido el 16 de junio de 2004; aceptado el 3 de enero de 2005
SARDELLA NH \& ME RE (1990) Parasitosis por coccidios del género Aggregata en pulpos costeros patagónicos. I. Aggregata sp. en Octopus tehuelchus D' Orbigny. Physis (Argentina) 46: 5160.

SARDELLA NH \& SR MARTORELLI (1997) Ocurrence of merogony of Aggregata Frenzel 1885 (Apicomplexa) in Pleoticus muelleri and Artemesia longinaris (Crustacea: Natantia) from Patagonian waters (Argentina). Journal of Invertebrate Pathology 70: 198-202.

SARDELLA NH, ME RE \& JT TIMI (2000) Two new Aggregata species (Apicomplexa: Aggregatidae) infecting Octopus tehuelchus and Enteroctopus megalocyathus (Mollusca: Octopodidae) in Patagonia, Argentina. Journal of Parasitology 86: 1107-1113.

VIDAL EAG \& M HAIMOVICI (1999) Digestive tract parasites in rhynchoteuthion squid paralarvae, particularly in Illex argentinus (Cephalopoda: Ommastrephidae). Fishery Bulletin 97: 402-405.

ZAR JH (1984) Biostatistical analysis. Second Edition. Prentice-Hall, Inc., New York, New York, USA. $622 \mathrm{pp}$. 